Introduction: Patients with cancer undergoing active systemic anticancer treatment (chemotherapy, immunotherapy, targeted, or combination therapy) are at greater risk of COVID-19 infection than persons without cancer. In this paper, the authors analyse the spread of the coronavirus among cancer patients undergoing systemic therapy, and the impact of COVID-19 infection on the continuation of cancer treatment and its outcome at one community hospital in a mid-sized city in the south of Poland. Material and methods: Nasopharyngeal swab was the only collection method used to obtain specimens for testing via real-time reverse-transcriptase polymerase chain reaction (RT-PCR). Only those with positive RT-PCR results were considered as confirmed SARS-CoV-2 cases. We analysed the medical records of patients quarantined in a hospital clinical oncology ward due to confirmed COVID-19 infection in one member of the group. Qualitative measures are presented as the percentage of their occurrence, and these were evaluated with Fisher's test. Differences were considered significant at $p<0.05$. Results: Cancer patients had more frequent confirmed COVID-19 infection than other patients $(3.7 \%$ vs. $1.2 \%)$. Among cancer patients COVID-19 infection was significantly more frequent in women than in men, $p=0.005$. The fatality rate was $27.3 \%$ in cancer patients undergoing active anticancer therapy, compared to $3 \%$ in the general Polish population. Neither heparin nor G-CSF use had any influence on COVID-19 infection. Conclusions: In this analysis, the only significant negative factor for COVID-19 infection was female sex, RR $(95 \% \mathrm{Cl})=4.5(1.3-15.8),(p=0.005)$, and this was attributable to individual behaviour.

Key words: coronavirus pandemic, COVID-19, cancer treatment, cancer patients, systemic therapy.

Contemp Oncol (Pozn) 2021; 25 (3): 147-152 DOI: https://doi.org/10.5114/wo.2021.109362

\section{Outcome of COVID-19 infection in cancer patients during active systemic anticancer treatment. Single-institution experience. A retrospective analysis}

\author{
Elżbieta Nowara', Eliza Działach², Mateusz Grajek², Zofia Kolosza³, \\ Joanna Huszno ${ }^{4}$
}

${ }^{1}$ Department of Health Sciences, Jan Dlugosz University of Humanities and Natural Sciences, Czestochowa, Poland

2Department of Public Health, Silesian Medical University, Katowice, Poland

${ }^{3}$ Narodowy Instytut Onkologii Gliwice Branch, Center for Translational Research and Molecular Biology of Cancer, Gliwice, Poland

${ }^{4}$ Department of Radiotherapy, Narodowy Instytut Onkologii Gliwice Branch, Gliwice, Poland

\section{Introduction}

Information about the rapid spread of the highly infectious SARS virus, which can cause a severe course of pneumonia, paralyzed the world. The World Health Organization's (WHO) declaration of a coronavirus pandemic prompted the authorities of most countries to react quickly to limit the spread of the disease. In Poland, a lockdown was announced at the beginning of March 2020. An order was issued for everyone to stay at home. Schools, offices, shops, and factories were closed. Hospitals were open only for emergency cases, with the exception of oncology departments.

Some authors claim that cancer patients, especially those undergoing active oncological treatment, have a higher risk of coronavirus infection and a poorer prognosis than patients without cancer. Most importantly, patients with cancer have been observed to have a higher risk of severe complications as compared with patients without. To the best of our knowledge, only a few case series or retrospective studies have been published to date, especially from China, where coronavirus infection was first found, and from Italy.

In this paper, the authors analyse the spread of the coronavirus among cancer patients, and the impact of COVID-19 infection on the continuation of cancer treatment and its outcome in one community hospital in a mid-sized city in the south of Poland.

To the best of our knowledge, this is one of the first reported clinical studies on clinical characteristics of cancer patients with SARS-CoV-2 infection in Poland.

\section{Material and methods}

In compliance with government recommendations in March 2020, all hospitals in Poland were closed for scheduled patients, and only emergency cases and cancer patients were admitted for necessary hospitalization. In our hospital, only the oncology and emergency wards remained open; other departments were prepared for the treatment of patients with COVID-19.

Nasopharyngeal or, in exceptional cases, pharyngeal swab was the only collection method used to obtain specimens for testing via real-time reverse-transcriptase polymerase chain reaction assay (RT-PCR), according to a validated protocol. As per WHO and Polish Government recommendations, those with positive RT-PCR results were considered as confirmed SARS-CoV-2 cases. 
Up until the time of closing the database, 1471 nasopharyngeal swabs were collected in our hospital including 55 cancer patients (March-April 2020). According to government recommendation, RT-PCR was performed in all patients who were admitted for hospital treatment. No one had symptoms of SARS-Cov-2 infection. Positive RT-PCR results were found in 25 persons ( $1.7 \%$ of all tests). Almost half of those (11 out of 25 patients - 44\%) were oncological patients undergoing active systemic treatment. So, 3.7\% of cancer patients had confirmed COVID-19 infection, compared to $1.2 \%$ of patients admitted to the hospital with other diseases.

The current retrospective analysis was performed on data from electronic records of cancer patients undergoing active systemic anticancer therapy in one of the community hospitals in a mid-sized city in the south of Poland. Active systemic anticancer treatment was defined as any anti-tumour systemic therapy (chemotherapy, immunotherapy, targeted, or combination therapy) administered after admission to the hospital.

Table 1. Clinical characteristics of quarantined patients

\begin{tabular}{|c|c|}
\hline $\begin{array}{l}\text { Total number of patients in quarantine } \\
\text { in a hospital ward }\end{array}$ & 20 \\
\hline Median age (range) & $66(21-76)$ \\
\hline Male/female & $10 / 10$ \\
\hline Median BMI (range) & $\begin{array}{c}25.05 \\
(19.4-35)\end{array}$ \\
\hline \multicolumn{2}{|l|}{ Cancer diagnosis } \\
\hline Gastrointestinal cancer & $8(40 \%)$ \\
\hline Lung cancer & $4(20 \%)$ \\
\hline Genitourinary cancer & $3(15 \%)$ \\
\hline Breast cancer & $2(10 \%)$ \\
\hline Gynaecological cancer & $2(10 \%)$ \\
\hline Head and neck cancer & $1(5 \%)$ \\
\hline \multicolumn{2}{|l|}{ Cancer stage/type of therapy } \\
\hline Local/adjuvant & $9(45 \%)$ \\
\hline Metastatic/palliative & $11(55 \%)$ \\
\hline Concomitant diseases & $14(70 \%)$ \\
\hline Metabolic diseases (e.g. diabetes) & $6(30 \%)$ \\
\hline $\begin{array}{l}\text { Cardiovascular system diseases (e.g. ischaemic } \\
\text { heart disease, hypertension) }\end{array}$ & $6(30 \%)$ \\
\hline Other cancers in the past & $2(10 \%)$ \\
\hline \multicolumn{2}{|l|}{ Additional prophylactic therapy } \\
\hline Heparin & $8(40 \%)$ \\
\hline G-CSF & $10(50 \%)$ \\
\hline COVID-19-infected patients & $11(55 \%)$ \\
\hline \multicolumn{2}{|l|}{ Symptoms and signs of COVID-19 infection } \\
\hline Fever & $2(10 \%)$ \\
\hline Dyspnoea & $2(10 \%)$ \\
\hline Fatigue & $1(5 \%)$ \\
\hline Asymptomatic liver insufficiency & $1(5 \%)$ \\
\hline
\end{tabular}

BMI - body mass index, G-CSF - granulocyte colony-stimulating factor
At the time of admission, all patients were evaluated based on Eastern Cooperative Oncology Group (ECOG) score, body mass index (BMI), concomitant diseases, concomitant medication, laboratory results, and clinical evaluation.

Only significant previous medical comorbidities were recorded: metabolic diseases (e.g. diabetes), cardiovascular system or heart diseases, and prevalence of other cancers in the past.

Previous anticoagulation therapy was identified in every patient under chronic anticoagulation with either lowmolecular-weight heparin $(\mathrm{LMWH})$ or oral anticoagulants.

We extracted an electronic medical record database of patients hospitalized in the Oncology Department, who had been quarantined due to contact on the ward with a patient who had tested positive for COVID-19.

Quarantine is the time of separation for person who is suspected to have had contact with another person with SARS-Cov-2 infection, and isolation is the time of separation for person who has had a positive result of a COVID-19 test. Both quarantine and isolation are obligatory by law.

The time of observation included the time of obligatory hospitalization in our department and the time to first control visit or the time of death.

Procedures were followed in accordance with the ethical standards of a responsible committee on human experimentation (institutional or regional) and within the terms of the Helsinki Declaration as revised in 2013.

\section{Patient characteristics}

We present the results of a retrospective, single-centre, observational study. We reviewed the records of $20 \mathrm{pa}$ tients admitted to the Clinical Oncology Department. During the hospitalization one of them was diagnosed with SARS-Cov-2 infection. According to Polish Government recommendations, all hospitalized patients had to be quarantined in the ward. All the patients had separated rooms with individual bathrooms.

All the patients with cancer were undergoing systemic anticancer treatment that required hospitalization.

As per PTOK (Polskie Towarzystwo Onkologii Klinicznej - Polish Society for Medical Oncology) recommendations, 10 patients (50\%) received granulocyte colony-stimulating factor (G-CSF) to protect against a nadir of inpatient neutropaenia, which could potentially result in sepsis [1]. G-CSF is a glycoprotein that stimulates the bone marrow to produce granulocytes and stem cells, and releases them into the bloodstream.

In our group, 8 patients were receiving chronic anticoagulation with $\mathrm{LMWH}$, as per PTOK recommendations.

The clinical characteristics are presented in Table 1.

\section{Statistical methods}

The authors analysed the available clinical, laboratory, and demographic data to detect features that could indicate predisposition amongst cancer patients to COVID-19 infection during active anticancer systemic treatment. We searched for any relationship by comparing these factors with the development of infection and the course 
of COVID-19 disease in these patients. We also analysed the neutrophil-to-lymphocyte ratio (NLR) and platelet-tolymphocyte ratio (PLR) to determine any impact on the risk of COVID-19 infection. The NLR is defined as the neutrophil count divided by the lymphocyte count. The PLR is defined as the platelet count divided by the lymphocyte count. Cut-off values were determined using receiver operating characteristic curves. The cut-off point for NLR was determined as elevated when $>4.83$, and the PLR was considered elevated when $>180.4$.

Statistical analysis was performed using Dell Statistica 13 software. Qualitative features were presented as the percentage of their occurrence and evaluated with Fisher's test. Differences were considered significant at $p<0.05$.

\section{Results}

The study group consisted of 20 patients: 10 women (50\%) and 10 men (50\%). The median age was 66 years (range 21-76). All patients were of Caucasian origin and were permanent residents of South Poland. All patients were in good general condition and had laboratory results within normal limits due to qualification for systemic treatment in a hospital setting. Gastrointestinal cancers (oesophagus, stomach, and colon cancer) were the most frequent in the study group $(n=8 ; 40 \%)$. The remaining patients had lung cancer (either non-small cell lung cancer, non-small cell lung carcinoma (NSCLC), or small cell lung cancer, small cell lung carcinoma (SCLC), $n=4 ; 20 \%$ ), cancers of the genitourinary system (testicular cancer or bladder cancer, $n=3 ; 15 \%)$, breast cancer ( $n=2 ; 10 \%)$, gynaecological cancer $(n=2 ; 10 \%)$, or head and neck cancer $(n=1 ; 5 \%)$. Eleven patients (55\%) had metastatic cancers, and 9 patients (45\%) were treated with adjuvant therapy. Seven patients received platinum derivative-based chemotherapy, and 4 had immunotherapy (trastuzumab, panitumumab, or pembrolizumab due to cancer type). Most patients (70\%) had concomitant diseases. During the time of observation, 11 patients (55\%) became infected with SARS-COV-2, and 6 of them (55\%) had clinical manifestation of infection, such as fiver, cough, dyspnoea, fatigue, and lose of taste and smell. Others had an asymptomatic course of disease, and one had asymptomatic liver insufficiency (AST, ALAT, GTTP many times above ULN). Nine patients did not become infected. No patients required intensive care unit support. In total, 9 of 10 women (90\%) and 2 of 10 men (20\%) developed COVID-19 infection.

The COVID-19 infection was significantly more frequent in women than in men, $\operatorname{RR}(95 \% \mathrm{Cl})=4.5(1.3-15.8)$, $(p=0.005)$ and insignificantly more common in the group of patients with comorbidities (64.3\% vs. 33.3\%; $p=0.336)$. Interestingly, there was no correlation between COVID-19 infection and cancer dissemination (45.5\% vs. $66.7 \%, p=0.406)$. COVID-19 infection was insignificantly more frequent in the group of patients with gastrointestinal cancers $(62.5 \%$ vs. $50 \% ; p=0.670)$ and insignificantly less frequent in patients with lung cancer (25\% vs. $62.5 \%$; $p=0.285)$. All women with gynaecological cancers tested positive for COVID-19, but no patient with genitourinary neoplasms became infected (0\% vs. 58.8\%; $p=0.074$ ).
Interestingly, LMWH use in a prophylactic setting did not have any influence on COVID-19 infection rates (50\% vs. $56.25 \%, p=1.0$ ). Similarly, use of G-CSF did not reduce the risk of infection ( $50 \%$ vs. $50 \% p=1.0$ ).

\section{Laboratory results}

There was no correlation between COVID-19 infection and increased leukocyte count above the ULN (50\% vs. $53.3 \% ; p=1.0)$. In patients with neutrophil count elevated above ULN, COVID-19 infection was observed insignificantly less frequently (33.3\% vs. $61.5 \%$; $p=0.350)$. There was no correlation between COVID-19 infection and increased lymphocyte counts (66.7\% vs. $50 \% ; p=1.00)$. In patients with thrombocytopaenia, COVID-19 infection was observed insignificantly more frequently $(66.7 \%$ vs. $50 \%$; $p=1.0)$. COVID-19 infection was observed only in patients with NLR $<4.83$ (62.5\% vs. 0\%; $p=0.087)$. Similarly, COVID-19 infection was not observed significantly more often in the group of patients with $\mathrm{PLR}<180.4$ (64.3\% vs. 20\%; $p=0.141)$.

Patients with elevated C-reactive protein (CRP) were insignificantly less likely to have COVID-19 infection (25\% vs. $75 \% ; p=0.065)$.

\section{Patient outcomes}

None of the patients were lost to follow-up. During the time period under observation, three patients with COVID-19 infection died (27.3\%). The median age at death was 64 years. All had comorbidities and metastatic cancers (gastric, SCLC, and colon) treated with palliative systemic treatment. Two died due to respiratory failure, and one due to multi-organ failure. The median time from COVID-19 diagnosis to death was 11 weeks.

Three patients were discharged in a stable condition from our hospital to others dedicated to the care of COVID-19 patients. Five others continued to quarantine at home until the date on which they obtained their third negative PCR result.

All patients' anticancer therapies were postponed until the end of their quarantine periods.

Careful observation of the behaviour of a group of patients prompts the authors to note the following insights: During their period of quarantine in the hospital ward, it was noted that men spent their time being truly isolated in their rooms, watching TV or playing computer games; however, women preferred to spend time together in the common room, talking and socializing, despite orders to stay separated in their rooms.

Detailed clinical negative factors for COVID-19 infection (factors favouring infection) are presented in Table 2.

\section{Discussion}

Cancer patients are believed to be at greater risk of COVID-19 infection because of their greater likelihood of being in an immunosuppressed state, resulting from both neoplasm and anticancer therapies [2, 3]. This is also the reason why cancer patients may be more susceptible to a more extreme course of the disease than the general population $[4,5]$. 
Table 2. Clinical negative factors for COVID-19 infection (factors favouring infection)

$\begin{array}{lcc}\text { Predictive factor } & p \text {-value } & \text { RR }(95 \% \mathrm{Cl}) \\ \text { Female sex } & 0.005 & 4.5(1.3-15.8) \\ \text { Comorbidities } & 0.336 & 1.9(0.6-6.4) \\ \text { BMI }>25 & 1.0 & 0.9(0.4-2.2) \\ \text { Cancer dissemination } & 0.406 & 0.7(0.3-1.5) \\ \text { Gastrointestinal cancer } & 0.670 & 1.3(0.6-2.7) \\ \text { Systemic therapy with } & 0.650 & 0.5(0.2-1.8) \\ \text { no platin derivatives } & & \\ \text { Leukocytosis } & 1.0 & 0.9(0.3-2.8) \\ \text { Thrombocytopaenia } & 1.0 & 1.3(0.5-3.4) \\ \text { NLR }<4.83 & 0.087 & 0.3(0.1-1.2) \\ \text { PLR < 180.4 } & 0.141 & 3.2(0.5-19.4) \\ \text { Elevated CRP in men } & 0.133 & 0.0\end{array}$

$B M I$ - body mass index, NLR - neutrophil-to-lymphocyte ratio, $P L R$ - platelet to-lymphocyte ratio, CRP - C - reactive protein

RT-PCR results described above revealed that that $44 \%$ of all positive tests performed in our hospital were obtained from cancer patients. After analysing the indications for the COVID-19 test (asymptomatic patients), we conclude that $3.7 \%$ of cancer patients in the hospital had a confirmed COVID-19 infection, compared to $1.2 \%$ of patients without any cancer diagnosis. By extrapolating from these data, it can be concluded that patients with malignant neoplasms undergoing active oncological treatment have a 3-fold higher risk of COVID-19 infection. These data are consistent with findings from others [4-6]. Our COVID-19 infected cancer patients were older (median 66 years) than the groups described by other European researchers $[7,8]$ but similar to those of Chinese publications $[2,9]$.

An interesting report from France included 15,600 breast cancer patients, of whom 59 patients were diagnosed with COVID-19. The authors examined the differences between patients with COVID-19 and those with no infection. They found that high BMI and hypertension did not increase the risk of COVID-19. Importantly, they found no trend in favour of a relationship between a history of breast and lymph node radiation therapy, radiation therapy sequelae, and radiologic extent of disease or outcome [10].

In this paper, an insignificantly lower risk of COVID-19 infection was seen in patients with genitourinary cancer ( 0 vs. $58.8 \%, p=0.074$ ). Additionally, patients with lung cancer did not have any increased risk for COVID-19 infection $(25 \%$ vs. $56.3 \%, p=0.285)$. In other studies, patients with lung cancer also did not have a higher probability of severe events as compared with patients with other cancer types [5].

In our paper, the only significantly negative factor was female $\operatorname{sex}(p=0.005)$, which was related to social behaviour.

The most common clinical symptoms of COVID-19 described in the literature include fever, cough, dyspnoea, fatigue, and - in rare cases - diarrhoea and vomiting [11]. In our group of COVID-19-infected cancer patients, only half had COVID-19 symptoms. The most frequent manifestations were fever and dyspnoea (10\% each), and fatigue and loss of taste and smell (10\% each). One person presented with asymptomatic liver insufficiency (AST, ALAT, GTTP many times above upper limit of the norm - ULN).

Some of the common laboratory findings were insignificantly more common in COVID-19 patients. Insignificantly higher risk of COVID-19 infection was noted in patients with an NLR $<4.83$ ( $p=0.087)$, and elevated CRP was another insignificantly positive prognostic factor $(p=0.065)$. Other studies have highlighted cytopaenias, specifically lymphocytopaenia, in COVID-19 patients [8, 11].

We also analysed the potential influence of using G-CSF on developing an infection. G-CSF is known as a cytokine commonly used in cancer patients receiving anticancer chemotherapy with high risk of severe neutropaenia, to help white blood cell levels recover after treatment. The main goal is to reduce the risk of febrile neutropaenia, allowing the patient to continue chemotherapy at the correct rhythm. There is evidence that G-CSF may lead to harmful clinical manifestation of COVID-19 infection, generally resulting in pulmonary immunopathology. This may be due to G-CSF strengthening pro-inflammatory cytokine expression due to promotion of differentiation of Th1 cells and polarization of macrophages [6]. In our analysis, G-CSF use did not have an influence on COVID-19 infection (50\% vs. $50 \% p=1.0$ )

The next topic of interest for the authors is whether the usage of low-molecular-weight heparin $(\mathrm{LMWH})$ may have an influence on COVID-19 infection and its course in cancer patients. The more that many cancer patients, especially with metastatic colorectal cancer, receive LMWH due to high risk of thromboembolism. It is known that LMWH has anti-inflammatory attributes and reduces the both biological activity and level of interleukin-6 (IL-6) [8]. IL-6, in turn, is produced by many types of white cells, including lymphocytes, monocytes, and fibroblasts. On the other hand, it is known that SARS-CoV-2 causes IL-6 production from bronchial epithelial cells [12-14]. That probably leads to a so-called 'cytokine storm', causing hypoxic respiratory failure [15-17]. In our analysis, low-molecular-weight LMWH use did not have an influence on COVID-19 infection $(p=1.0)$

Some authors claim that the course and severity of symptoms of COVID-19 may be different in cancer patients than in the general population. In cancer patients, the mean time from the onset of symptoms until respiratory failure is 7.02 days amongst hospitalized non-cancer patients [15]; unfortunately, the mean time to death in our study was shorter (12.4 days in contrast to 21.0). These data may suggest that cancer patients have a more rapid COVID-19 infection course in comparison to others.

In this study, over the time of observation, $27.3 \%$ of cancer patients with COVID-19 infection died at a median age of 64 years. The median time from COVID-19 diagnosis to death was 11 weeks. At the time of writing, based on the reports of the Polish Ministry of Health, the mortality rate in the general Polish population is 3\% [16]. A similar fatality rate for infected cancer patients was reported in China: $28.6 \%$ [17], compared to a $2.3 \%$ fatality rate for all 
COVID-19 patients $[18,19]$. However, others claim that the fatality rate may be only as high as 5.6\% [20].

To date, there is still insufficient information about the treatment of COVID-19 in cancer patients because of the small number of sample cases, observational trials, high heterogeneity of the courses of neoplasm diseases, and lack of prospective trials.

There is still an urgent need to answer the question of whether cancer patients really undergo a more damaging course of SARS-CoV-2 infection, and whether there is the safe possibility of continuing anticancer treatment during infection [20]. In our group of patients, 2 died due to respiratory failure, and 1 due to multi-organ failure. It is difficult, however, to conclude whether these deaths were due to their interrupted cancer treatments or due to COVID-19 infection.

Among cancer patients, the probability of COVID-19 infection may be even lower due to a much stricter application of social distancing procedures, after being informed that they may be at higher risk of severe COVID-19 symptoms. Prophylactic changes implemented in cancer care (e.g. postponement of all non-mandatory visits to $\mathrm{ICH}$, changes in medical treatments) may also have contributed to further reducing the risk of SARS-CoV-2 infection [9]. Unfortunately, many of cancer patients may also give up their treatment due to serious fear of infection.

The Center for Disease Control and Prevention guidelines call for wearing a mask that covers both your nose and your mouth, with the mask secured under your chin. It should fit snugly against your face. There should not be large openings or gaps around your nose, mouth, or the sides of your face [21].

Clear communication and education about the following should all be reinforced: hand hygiene; infection control measures; the signs and symptoms of COVID-19; highrisk travel or exposure; and the importance of reporting new symptoms to health care workers [13].

Our study has several limitations. The biggest one is that the study was a retrospective, single institution study with small sample size. Furthermore, the study was with incomplete clinical data.

The authors emphasize that none of the staff caring for these quarantined patients were infected with COVID-19. All staff scrupulously followed the rules regarding the wearing of personal protective equipment.

The authors of the current paper believe that social distancing and the wearing of personal masks in the correct way are the most important factors to avoid COVID-19 infection.

\section{Conclusions}

In this paper, the only significantly negative factor for COVID-19 infection was female sex $(p=0.005)$, probably due to social behaviour. Furthermore, insignificantly higher risk of COVID-19 infection was seen in patients with an NLR $<4.84(p=0.0877)$, while insignificantly lower risk of COVID-19 infection was seen in patients with genitourinary tract cancer and CRP elevation ( $p=0.074$ and $p=0.065$, respectively). Use of G-CSF did not reduce the risk of infection $(p=1.0)$. The fatality rate was $27.3 \%$ in cancer patients undergoing active anticancer therapy, compared to $3 \%$ in the general Polish population.

The authors express the opinion that the social behaviour of patients, their families, and medical staff may have the greatest impact on COVID-19 infections. This is due to the observation that, in our group of patients, women were willing to meet outside of their rooms and spend time chatting together. The men, on the other hand, spent their quarantine in their rooms, isolating themselves from each other, watching TV, and using their personal computers.

\section{The authors declare no conflict of interest.}

\section{References}

1. Wysocki PJ, Kwinta L, Potocki P, et al. Leczenie systemowe pacjentów z rozpoznaniem choroby nowotworowej w kontekście pandemii SARS-CoV-2 - stanowisko Polskiego Towarzystwa Onkologii Klinicznej. Onkol Prakt Klin Edu 2020; 7: 131-135.

2. Yarza R, Bover M, Paredes D, et al. SARS-CoV-2 infection in cancer patients undergoing active treatment: analysis of clinical features and predictive factors for severe respiratory failure and death. Eur J Cancer 2020; 135: 242-250.

3. Liang W, Guan W, Chen R, et al. Cancer patients in SARS-CoV-2 infection: a nationwide analysis in China. Lancet Oncol 2020; 21 : 335-337.

4. Chavez S, Long B, Koyfman A, Liang SY. Coronavirus Disease (COVID-19): a primer for emergency physicians. Am J Emerg Med 2020; S0735-6757(20)30178-9.

5. Liang W, Guan W, Chen R, et al. Cancer patients in SARS-CoV-2 infection: a nationwide analysis in China. Lancet Oncol 2020; 21: 335-337.

6. Gosain R, Abdou Y, Singh A, Rana N, Puzanov I, Ernstoff MS. COVID-19 and Cancer: a Comprehensive Review. Curr Oncol Rep 2020; 22: 53.

7. World Health Organization. WHO clinical management of severe acute respiratory infection (SARI) when covid-19 disease is suspected. WHO 2020.

8. Qian Y, Xie H, Tian R, Yu K, Wang R. Efficacy of low molecular weight heparin in patients with acute exacerbation of chronic obstructive pulmonary disease receiving ventilatory suport. Randomized controlled trial. J Chronic Obstr Pulm Dis 2014; 11: 171-176.

9. Dai M, Liu D, Liu M, et al. Patients with Cancer Appear More Vulnerable to SARS-COV-2: a multicenter study during the COVID-19 outbreak. Cancer Discov 2020; 10: 783-791.

10. Vuagnat P, Frelaut M, Ramtohul T, et al. COVID-19 in breast cancer patients: a cohort at the Institut Curie hospitals in the Paris area. Institut Curie Breast Cancer and COVID Group, Bozec L, Paoletti X, Cottu P, Bidard FC. Breast Cancer Res 2020; 22: 55.

11. Lippi G, Mattiuzzi C, Sanchis-Gomar F, Henry BM. Clinical and demographic characteristics of patients dying from COVID-19 in Italy vs. China. J Med Virol 2020; 10: 1002/jmv.25860.

12. Zhang H, Xie C, Huang Y. Treatment and outcome of a patient with lung cancer infected with severe acute respiratory syndrome coronavirus. J Thorac Oncol 2020; 15: e-63-e63.

13. Al-Shamsi HO, Alhazzani W, Alhuraiji A. A practical approach to the management of cancer patients during the novel coronavirus disease 2019 (COVID-19) pandemic: an international collaborative group. Oncologist 2020; 25: e936-e945.

14. Aggarwal S, Garcia-Telles N, Aggarwal G, Lavie C, Lippi G. Clinical features, laboratory characteristics, and outcomes of patients hospitalized with coronavirus disease 2019 (COVID-19): early report from the United States. Diagnosis (Berl) 2020; 7: 91-96.

15. Zhou F, Yu T, Du R, et al. Clinical course and risk factors for mortality of adult inpatients with COVID-19 in Wuhan, China: a retrospective cohort study. Lancet 2020; 395: 1054-1062. 
16. https://www.gov.pl/web/koronawirus

17. Zhang L, Zhu F, Xie L, et al. Review of the 2019 novel coronavirus (SARS-CoV-2) based on current evidence. Int J Antimicrob Agents 2020; 55: 105948

18. Wang L, Wang Y, Ye D, Liu Q. Clinical characteristics of COVID-19 infected cancer patients: a retrospective case study in three hospitals within Wuhan, China. Ann Oncol 2020; 31: 894-901.

19. Pathania AS, Prathipati P, Abdul BA, et al. COVID-19 and cancer comorbidity: therapeutic opportunities and challenges. Theranostics 2021; 11: 731-753.

20. Palmieri L, Vanacore N, Donfrancesco C, et al. Clinical characteris tics of hospitalized individuals dying with COVID-19 by age group in italy. J Gerontol A Biol Sci Med Sci 2020; 75: 1796-1800.

21. https://www.cdc.gov/.

\section{Address for correspondence}

\section{Elżbieta Nowara}

Wydział Nauk o Zdrowiu

Uniwersytet Humanistyczno-Przyrodniczy Jana Długosza

Częstochowa, Polska

e-mail: elzbieta.nowara@interia.pl

Submitted: 16.02 .2021

Accepted: 05.07.2021 